\title{
Estudio cognitivo sobre el contenido de conceptos (científicos, concretos y abstractos) y la influencia del conocimiento
}

\author{
Cognitive Study on the Content of Concepts (Scientific, \\ Concrete and Abstract) and the Influence of Knowledge
}

\author{
Geral Eduardo Mateus Ferro ${ }^{1}$
}

\section{Resumen}

Este artículo tiene como objetivo hacer un estudio cognitivo sobre el contenido de conceptos científicos, concretos y abstractos en 139 participantes distribuidos en tres grupos con distinto conocimiento en ciencias (alto, medio y bajo). La metodología se centra en la realización de tareas de generación de propiedades y el posterior análisis de los protocolos de respuesta según el modelo planteado por Wu y Barsalou (2009). Se analiza si las diferencias de conocimiento entre los grupos de participantes inciden en la manera como se almacenan esta clase de conceptos en la memoria. Los resultados estadísticos revelan diferencias en las propiedades que conforman esta clase de conceptos, al igual que otras atribuibles a la variable conocimiento, especialmente en los conceptos científicos

\section{Palabras clave}

Contenido conceptual, tarea de generación de propiedades, cognición, cognición situada, análisis de protocolos.

\section{Abstract}

The study attempts to examine memory content of scientific, concrete and abstract concepts in 139 participants. They differ in their science knowledge (high, medium and low). Property generation task and protocol analysis from Wu and Barsalou's model (2009) are the methodology base. Knowledge differences in memory content in those groups are analyzed. Statistics reveal that concepts properties differ and that other differences between concepts are due to knowledge as a study variable, primarily in scientific concepts.

\section{Keywords}

Conceptual content, property generation tasks, cognition, situated cognition, protocol analysis.

Artículo recibido el 11 de abril de 2014 y aprobado el 20 de agosto de 2014

1 Universidad Pedagógica Nacional, Bogotá, Colombia. Correo electrónico: gmateus@pedagogica.edu.co 


\section{Introducción}

En el marco de los estudios sobre los conceptos, desde la psicología cognitiva se han adelantado investigaciones con el objetivo de determinar el contenido de los mismos en términos de propiedades. ${ }^{2}$ En tal sentido, a través de tareas de generación de propiedades se ha logrado determinar qué clase de información integra los conceptos, teniendo en cuenta de manera especial la distinción entre conceptos concretos y abstractos (Barsalou y Wiemer-Hastings, 2005; Cree y McRae, 2003; McRae y Cree, 2002; McRae, de Sa y Seidenberg, 1997; Santos, A., Chaigneau, S., Simmons, W. y Barsalou, L., 2011; Wu y Barsalou, 2009).

Desde la perspectiva de las teorías corpóreas, Barsalou y sus colegas (Barsalou y Wiemer-Hastings, 2005; Santos et al., 2011; Wiemer-Hastings y Xu, 2005; Wu y Barsalou, 2009) realizaron un conjunto de investigaciones para probar que en el proceso de simulación de los conceptos intervienen varios tipos de conocimiento (propiedades). A través del análisis de protocolos de generación de propiedades, Wu y Barsalou (2009) estudiaron el contenido conceptual de conceptos concretos. Se pedía a los participantes que dijeran, inmediatamente después de la presentación de un concepto diana, las propiedades que creyeran pertenecían a dicho concepto. Se asume que las propiedades generadas en la tarea provienen de la simulación que surge en la mente de los participantes después de haber recibido el estímulo lingüístico. Tras el análisis de las propiedades producidas se halló que en dichos conceptos los participantes no solamente incluían propiedades relativas a características de la entidad y a su clase taxonómica, sino que también presentaban propiedades y elementos de las situaciones en las cuales

2 Desde un punto de vista más filosófico que psicológico, Field (2008) resalta dos sentidos del término propiedad: propiedad natural $y$ propiedad conceptual. En el sentido de propiedad natural se hace referencia a las cualidades de las entidades (p.ej., mamífero, tímido, azul). El otro sentido de propiedad, propiedad conceptual, alude a las entidades mentales que se corresponden con los predicados sobre cualidades de las entidades. Este segundo sentido, feature (en inglés), es el más extendido en el ámbito psicológico, el cual define el conjunto de conocimientos (entidades mentales) que constituyen un concepto dado. En este artículo se opta por esta última acepción de propiedad. los objetos suelen aparecer, así como apreciaciones subjetivas (introspecciones) de su experiencia con estos objetos. Por ejemplo, para el concepto manzana los participantes generan propiedades relativas al tamaño, color, forma, sabor, es decir, propiedades de la entidad misma, otras taxonómicas (p. ej., "es una fruta, un vegetal"), así como referencias a las situaciones y elementos junto con los que se encuentran los melones (p. ej., "se comen en el postre, se cortan con cuchillos"), y a su experiencia personal, incluso "mental", con el concepto (p. ej., "me gustan las verdes").

Para medir el contenido de los conceptos, $\mathrm{Wu}$ y Barsalou (2009) diseñaron un modelo de codificación de las propiedades (en adelante modelo $\mathrm{wb}$ ) con cinco categorías generales (propiedades taxonómicas, de entidad, situacionales, introspectivas y miscelánea), las cuales, a su vez, incluían niveles más específicos de codificación (una versión completa y actualizada del modelo puede consultarse en Mateus y Otero, 2011). Las propiedades taxonómicas $(T)$, por ejemplo, recogen las menciones a las categorías supraordenadas o subordinadas al concepto diana; bajo la categoría de entidad $(E)$ se codifican las características del concepto, así como sus funciones; las propiedades situacionales $(S)$ contemplan los eventos, las situaciones y elementos incluidos en estas; en la categoría de introspección $(I)$ se sitúan las referencias al estado mental, a las operaciones cognitivas del sujeto, así como a las evaluaciones afectivas en relación con el concepto diana; en el grupo de miscelánea $(M)$ se codificaron las informaciones sin relevancia teórica para esta clase de estudios (p. ej., repeticiones, vacilaciones, metacomentarios sobre la tarea, etc.).

Wu y Barsalou (2009) hallaron que la mayoría de las propiedades que se generan en conceptos concretos son propiedades de la entidad. Sin embargo, el $26 \%$ del total de características expresadas corresponden a propiedades de la situación e introspecciones. Este resultado respalda parte de las tesis de las teorías corpóreas, especialmente en lo referente a que en el proceso de comprensión de un término $\mathrm{y}$ el concepto asociado, junto al procesamiento puramente lingüístico de las palabras, se produce 
una simulación que incluye tanto información abstracta y general como información flexible proveniente de la percepción, las distintas modalidades sensoriales y la experiencia del participante con dicho concepto (Barsalou, 1999; Glenberg, 2007; Glenberg y Kaschak, 2002; Glenberg y Robertson, 2000; Santos et al., 2011; Yeh y Barsalou, 2006; Wu y Barsalou, 2009).

El modelo wb también fue empleado con algunas modificaciones, para medir el contenido conceptual de conceptos concretos en otras investigaciones (Barsalou y Wiemer-Hastings, 2005; Cree y McRae, 2003; McRae y Cree, 2002; McRae, Cree, Seidenberg y McNorgan, 2005). Con base en los datos de un estudio normativo previo, McRae et al., (1997; McRae y Cree 2002; Cree y McRae, 2003) analizaron el contenido de 549 conceptos concretos utilizando el modelo wb. El reparto de propiedades generadas, al igual que en el trabajo de Wu y Barsalou (2009), mostró una mayor proporción de propiedades de entidad, seguido por las situacionales, taxonómicas e introspectivas. Es decir, la mayor parte de las propiedades generadas en conceptos concretos corresponden a las características de la entidad.

No obstante, Barsalou y Wiemer-Hastings (2005) obtuvieron resultados contradictorios con los de los estudios anteriores. A pesar de que el modelo wb se diseñó para el estudio de materiales concretos, Barsalou y Wiemer-Hastings (2005) lo aplicaron también, con algunas modificaciones, en el análisis de propiedades generadas para conceptos abstractos como verdad, invención o libertad. Esta vez los resultados arrojaron que la mayor parte de las propiedades generadas en los conceptos abstractos fueron las de situación.

En resumen, los datos de las investigaciones sobre generación de propiedades que utilizan el modelo wb indican que todos los conceptos integran propiedades pertenecientes a las entidades mismas con propiedades relacionadas con las situaciones y experiencias en que suelen aparecer esas entidades. Sin embargo, un resultado fundamental es que las referencias a propiedades de entidad son mayores en los conceptos concretos que en los conceptos abstractos, en tanto que las propiedades situacionales e introspectivas son mayores en los segundos que en los primeros.

\section{Conceptos científicos}

Los filósofos y sociólogos de la ciencia, especialmente, al igual que los propios científicos, han intentado establecer los límites entre aquellas actividades intelectuales que se consideran ciencia de aquellas que no lo son (cfr. Giergyn, 1983). Una vía de análisis se ha centrado en la especificidad de los conceptos manejados por la ciencia y en sus posibles clasificaciones (cfr. en filosofía de la ciencia, Achinstein, 1968; Hempel, 1952; Suppe, 1973/1990; Wartofsky, 1976; en ciencias, Chi, 1992, 2005; Chi, Slotta y de Leeuw, 1994; Reiner, Slota, Chi y Resnik, 2000; Lawson, Alkhouty, Benford, Clark y Falconer, 2000; Reif, 1987; Riefy Allen; 1992). También, a partir de las clasificaciones de conceptos construidas desde la psicología, ha podido vislumbrarse el carácter "especial" de los conceptos científicos (cfr. Keil, 1989, Murphy y Medin, 1985) frente a los demás. En general, se considera que los conceptos construidos por la ciencia corresponden a una clase "especial" dentro de los conceptos abstractos, dado su carácter general y su imposibilidad de percepción directa, pues muchos de ellos corresponden a entidades teóricas.

En un intento de responder a la pregunta, Mateus y Otero (2011) emplearon el modelo wb para estudiar el contenido conceptual de conceptos científicos, hallando que más de la mitad de las propiedades generadas fueron las de entidad, seguidas por las situacionales, taxonómicas e introspectivas, cuyas proporciones no superaron el $18 \%$.

Este estudio tiene como objetivo contrastar el contenido conceptual de conceptos científicos, abstractos y concretos. Para tal fin se plantea un estudio en el cual se emplea la generación de propiedades para estos tres tipos de conceptos. Adicionalmente, se estudia la influencia de la variable conocimiento para analizar su incidencia en la simulación de dichos conceptos y, consecuentemente, en su contenido conceptual. Dada la especificidad reconocida del conocimiento científico se tomó este como punto de referencia para establecer los parámetros de la variable conocimiento. 
Para establecer las diferencias de conocimiento en ciencias se tomó como punto de partida la formación y experiencia específica: los participantes graduados de estudios universitarios en ciencias y experiencia en investigación se consideran de alto conocimiento; los que poseen estudios universitarios en el área sin obtener el grado y no poseen experiencia investigativa son los de conocimiento medio; aquellos participantes cuya formación en ciencias se restringe a la recibida en la educación media, son los de bajo conocimiento.

En términos generales, en lo que comporta a la naturaleza de los conceptos, se espera que los tres tipos de conceptos analizados - científicos, concretos y abstractos- difieran en su contenido. Específicamente, se espera confirmar los hallazgos de Mateus y Otero (2011), en cuanto se espera un mayor foco de la simulación de los conceptos científicos en las propiedades de entidad, al igual que en los conceptos concretos no científicos. Sin embargo, también se espera que en estos últimos haya menos propiedades de entidad y más situacionales que en los científicos. Respecto a los conceptos abstractos, se supone que su foco de simulación sean las propiedades situacionales e introspectivas. En consecuencia, se espera que haya diferencias en el carácter situado entre los conceptos. La revisión de los antecedentes sugeriría que los conceptos científicos son los menos situados, es decir, los que presentarían menos propiedades situacionales e introspectivas que los concretos y los abstractos no científicos.

Por otra parte, es de esperar que las diferencias de conocimiento entre los participantes se relacionen con repartos diferenciales de propiedades en los conceptos científicos. En cuanto que no se esperan diferencias en el reparto de propiedades de los conceptos concretos y abstractos asociados con la variable conocimiento científico de los sujetos. Si las diferencias de reparto tuvieran lugar efectivamente solo en los conceptos científicos, indicaría que la formación en ciencias tiene cierto tipo de influencia en la simulación de los conceptos que maneja, pero no en conceptos de otros dominios.

\section{Método}

\section{Participantes}

Tomaron parte en el experimento 139 participantes con distintos conocimientos en ciencias: 59 estudiantes de licenciatura en español y lenguas extranjeras, 51 estudiantes de la licenciatura en diseño tecnológico y 29 profesionales universitarios graduados en ciencias (tabla 1).

Tabla 1. Distribución de los participantes

\begin{tabular}{|c|c|c|}
\hline Conocimiento & Número de participantes & \begin{tabular}{c} 
Formación/experiencia en ciencias \\
\hline Alto
\end{tabular} \\
\hline Medio & 51 & $\begin{array}{l}\text { • Licenciatura y/o posgrado en ciencias } \\
\text { • Docencia e investigación en ciencias experimentales. }\end{array}$ \\
\hline Bajo & 59 & • Dos cursos universitarios de pregrado en física \\
\hline
\end{tabular}




\section{Materiales y diseño}

Se estudiaron 20 conceptos: 12 conceptos científicos, 4 conceptos no científicos concretos y 4 conceptos no científicos abstractos.

En la selección de los conceptos científicos se tuvo en cuenta que fuesen representativos de las tres principales clases de conceptos científicos: objetos, procesos y propiedades (Chi, 1992, 2005, 2008; Chi, Slotta y de Leeuw, 1994). Con base en el procedimiento de Mateus y Otero (2011), se comprobó que los conceptos tuvieran un alto porcentaje de frecuencia en el área de ciencias en el Corpus 92: el $84 \%$ de las apariciones de los 12 conceptos elegidos tiene lugar exclusivamente en el área de ciencias (tabla 2).

Tabla 2. Listado de conceptos científicos

\begin{tabular}{|c|c|c|}
\hline \multicolumn{3}{|c|}{ Conceptos científicos } \\
\hline Objetos & Procesos & Propiedades \\
\hline Molécula & Ebullición & Temperatura \\
\hline Electrón & Evaporación & Densidad \\
\hline Resistor & Refracción & Velocidad \\
\hline Batería & Reflexión & Aceleración \\
\hline
\end{tabular}

Los 8 conceptos no científicos, 4 concretos y 4 abstractos (tabla 3), se eligieron de los materiales empleados en dos estudios previos sobre el contenido conceptual y generación de propiedades (Barsalou y Wiemer-Hastings, 2005; WiemerHastings y Xu, 2005). El grado de concreción de dichos conceptos fue verificado con las bases de datos del LEXESP-CORCO (Sebastián, Carreiras, Cuetos y Martí, 2000). En la escala de concreción del Lexesp (que va de 1 a 7, donde 7 estima el máximo grado de concreción), el promedio de los conceptos concretos seleccionados es de 6,02, en tanto que el de los conceptos abstractos es de 2,87.
Tabla 3. Conceptos no cientíicos concretos y abstractos

\begin{tabular}{|c|c|}
\hline \multicolumn{2}{|c|}{ Conceptos no científicos } \\
\hline Concretos & Abstractos \\
\hline Árbol & Verdad \\
\hline Sofá & Felicidad \\
\hline Laberinto & Esperanza \\
\hline Lazo & Aspecto \\
\hline
\end{tabular}

Se diseñaron 12 cuadernillos que contenían 10 conceptos distribuidos en dos grupos: 6 conceptos científicos ( 2 objetos, 2 procesos y 2 propiedades) y 4 conceptos no científicos ( 2 concretos y 2 abstractos). El orden de los conceptos en cada grupo era aleatorio.

Todos los participantes generaron propiedades para 10 conceptos ( 4 científicos, 2 concretos y 2 abstractos). En total se generaron 1390 protocolos de respuesta (139 participantes $\times 10$ conceptos).

El diseño definitivo comprende una variable intrasujetos, tipo de concepto (científico, concreto, abstracto) y otra intersujetos, conocimiento (alto, medio, bajo), medidas en los cuatro principales tipos de propiedad del modelo wb (T, E, S, I).

\section{Procedimiento y análisis}

En el caso de los grupos de conocimiento bajo y medio, la prueba se llevó a cabo en una hora corriente de clase; en el caso del grupo de conocimiento alto, la prueba se realizó en sesiones individuales. En los dos casos las aplicaciones tomaron aproximadamente 40 minutos.

Dado que había conceptos científicos y no científicos en el mismo cuadernillo, este tenía dos partes, con instrucciones semejantes, excepto por la mención en las instrucciones de la segunda parte de que en ese apartado encontrarían conceptos científicos. Todos los participantes generaron primero las propiedades para los conceptos científicos y luego para los no científicos. No se mezclaron para evitar interferencias entre los tipos de conceptos. 
Los cuadernillos se distribuyeron aleatoriamente. En la primera página del cuadernillo estaba escrita la siguiente instrucción, la cual fue leída en voz alta por un investigador antes de la prueba:

El propósito general de esta prueba es estudiar el conocimiento sobre algunos conceptos. Antes de comenzar es preciso resaltar que no hay respuestas correctas predeterminadas a las preguntas que haremos. Así que no se preocupe si no encuentra las respuestas. Esta prueba hace parte de un estudio cuyo propósito es comprender cómo los estudiantes generan pensamientos sobre algunos conceptos.

Cuando se pregunte por los conceptos, por favor escriba los primeros pensamientos que vengan a su mente y continúe escribiendo los pensamientos hasta cuando se le pida que se detenga.

Después de leer las instrucciones se hicieron dos ensayos de generación de propiedades por escrito con dos conceptos distintos de los elegidos (p. ej., balón y masa). La instrucción exacta era:

Por favor escriba, tal como se generen en su mente, la mayor cantidad de características e ideas sobre el siguiente concepto: [nombre del concepto].

Enseguida los participantes escribían las propiedades. Se les explicó que las ideas se parecen más a un texto que a un listado de términos, por esta razón los resultados no corresponden a listados de propiedades sino a textos con ideas desarrolladas en oraciones.

Posteriormente se inició la prueba. Después de leer la instrucción, los estudiantes escribían las propiedades del concepto correspondiente durante dos minutos. (A diferencia de las investigaciones previas en las cuales los participantes generaban en voz alta las propiedades durante un minuto, al llevarse a cabo la prueba por escrito se permitió más tiempo para la realización de la tarea). Enseguida se descansaba al menos durante un minuto y se continuaba con el siguiente concepto.
Finalizada la tarea sobre cada concepto, se revisaba que la prueba fuera en orden y que los estudiantes no volvieran sobre los ítems resueltos o que hojearan los siguientes. Tampoco se les permitía hablar sobre el concepto que estaban escribiendo. Al terminar se recogieron los cuadernillos y se revisó que todos estuviesen debidamente rellenos.

\section{Análisis}

Cada uno de los 1390 protocolos fue transcrito en un procesador electrónico de texto. Posteriormente fue analizado y segmentado en sus propiedades, asignándole una categoría ( $T, E, S, I, M)$ del modelo wb por parte de un solo codificador. La segmentación no siempre correspondió a una simple división de los protocolos palabra por palabra, sino que en algunos casos fue necesario dejar en un segmento más de una palabra porque de lo contrario la unidad quedaría incompleta. Por ejemplo, la expresión de 5 palabras "los seres humanos necesitan energía", no comprende 5 unidades sino tres, (1) "los seres humanos", (2) "necesitan" y (3) "energía”.

Una muestra del $5 \%$ de los protocolos de codificación fue sometida a una prueba de acuerdo con cuatro jueces más (dos profesores universitarios y dos licenciados, todos con experiencias en ciencias). El coeficiente kappa entre las cinco codificaciones fue $k=0,74$. Los desacuerdos se resolvieron mediante discusión entre los codificadores.

La tabla 4 muestra un ejemplo de la codificación de las propiedades en un protocolo. 
Tabla 4. Protocolo analizado para el concepto temperatura

\begin{tabular}{|c|c|l||}
\hline Posición & Análisis 1 & \multicolumn{1}{c|}{ Protocolo } \\
\hline 1 & T & (Es) la magnitud \\
\hline 2 & M & Que \\
\hline 3 & E & se encarga de medir \\
\hline 4 & E & el tiempo meteorológico \\
\hline 5 & S & que hace en un momento determinado. \\
\hline 6 & M & Normalmente \\
\hline 7 & M & se mide \\
\hline 8 & E & en grados centígrados \\
\hline 9 & aunque también pueden ser \\
\hline 10 & M & kelvin. \\
\hline 11 & M & se mide magnitud \\
\hline 12 & S & con los termómetros. \\
\hline 13 & E & Hay diferentes \\
\hline 14 & M & Temperaturas \\
\hline 15 & T & (como) la corporal, \\
\hline 16 & T & la meteorológica. \\
\hline & & \\
\hline
\end{tabular}

\section{Resultados}

La proporción de cada tipo de propiedad generada en los tres tipos de conceptos por cada participante fue transformada a su arcoseno para normalizar su varianza. Al conjunto de datos se aplicó un Anova para muestras repetidas para los factores tipo de concepto y conocimiento, posteriormente se aplicaron pruebas post hoc para comparar las diferencias específicas en cada factor y en las interacciones estadísticamente significativas (tal como se mencionó, las propiedades codificadas en la categoría miscelánea, dada su irrelevancia teórica, no se tuvieron en cuenta en el análisis). La tabla 5 presenta la proporción de las cuatro propiedades generadas para los tres tipos de conceptos discriminando los tres grupos de conocimiento.

Tabla 5. Proporciones de las propiedades generadas para los tres conceptos en los tres niveles del conocimiento y promedios generales por tipo de concepto

\begin{tabular}{|c|c|c|c|c|c|c|c|c|c|c|c|c|c|}
\hline \multirow{7}{*}{ 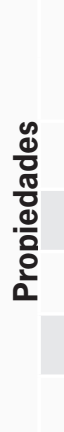 } & & \multicolumn{12}{|c|}{ TIPOS DE CONCEPTO } \\
\hline & & \multicolumn{4}{|c|}{ Científicos Promedio } & \multicolumn{4}{|c|}{ Concretos Promedio } & \multicolumn{4}{|c|}{ Abstractos Promedio } \\
\hline & Conocimiento & Alto & Medio & Bajo & & Alto & Medio & Bajo & & Alto & Medio & Bajo & \\
\hline & Taxonómicas & .08 & .09 & .10 & .09 & .08 & .10 & .10 & .09 & .07 & .12 & .12 & .11 \\
\hline & Entidad & .93 & .81 & .71 & .79 & .57 & .60 & .59 & .59 & .17 & .36 & .44 & .35 \\
\hline & Situacionales & .07 & .15 & .21 & .15 & .28 & .27 & .29 & .28 & .39 & .28 & .26 & .30 \\
\hline & Introspectivas & .06 & .05 & .05 & .05 & .12 & .08 & .07 & .09 & .38 & .27 & .21 & .26 \\
\hline
\end{tabular}

Nota: La suma de las proporciones no es 1, debido a la transformación arcoseno de las mismas. 
Las pruebas estadísticas arrojaron un efecto significativo del factor intrasujetos, concepto $(\mathrm{F}(8$, $129)=101.742, \mathrm{p}<.001)$ y de la variable intersujetos conocimiento $(\mathrm{F}(8,268)=10.324, \mathrm{p}<.001)$. También es significativa la interacción entre los dos factores $(\mathrm{F}(16,260)=5.066, \mathrm{p}<.001)$.

La variable dependiente propiedad también presenta efecto principal $(\mathrm{F}(3,134)=1540.048$, $\mathrm{p}<.001)$, e interactúa tanto con la variable conocimiento $(\mathrm{F}(6,270)=6.279, \mathrm{p}<.001)$, como con la variable concepto $(\mathrm{F}(6,131)=126.640, \mathrm{p}<.001)$. Adicionalmente, se presenta interacción entre los tres factores propiedad, concepto y conocimiento $(\mathrm{F}(12,164)=5.980, \mathrm{p}<.001)$.

En la siguiente presentación detallada de los resultados se describen las diferencias en cada propiedad ( $T, E, S, I$ ), en los dos factores (concepto y conocimiento). La figura 1 ilustra los resultados de la tabla 5 .

Figura 1. Proporciones de las propiedades por concepto y conocimiento

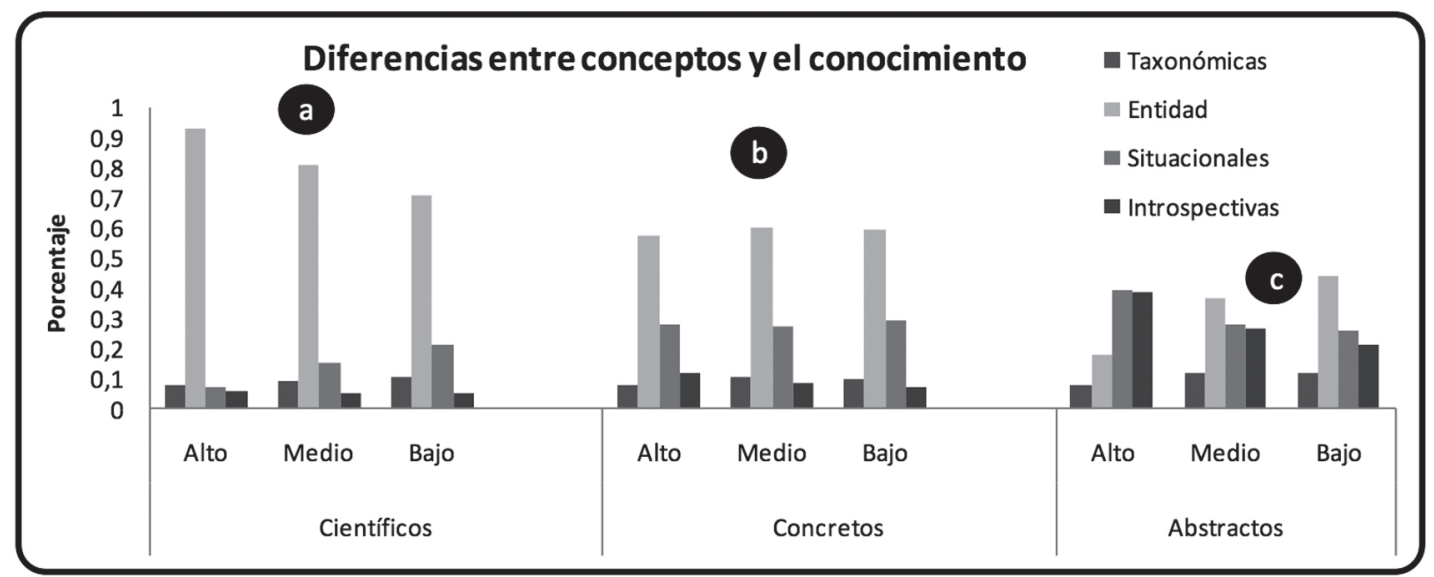

\section{Propiedades taxonómicas}

Los análisis de los datos de las propiedades taxonómicas no arrojaron efecto principal del factor concepto $(\mathrm{F}(2,135)=1.857 \mathrm{p}>.05)$, ni interacción concepto $\times$ conocimiento $(\mathrm{F}(4,272)=1.074, \mathrm{p}>.05)$.

\section{Propiedades de entidad}

Las pruebas estadísticas de las propiedades de entidad revelan efecto principal del factor concepto $(\mathrm{F}(2$, $135)=283.462, \mathrm{p}<.001)$, e interacción concepto $\times$ conocimiento $(\mathrm{F}(4,272)=16.512, \mathrm{p}<.001)$.

Diferencias entre conceptos. La pruebas post hoc de los porcentajes de propiedades de entidad en los conceptos científicos (figura 1, sección a) muestran que los participantes de conocimiento alto generan estadísticamente más propiedades que los de conocimiento medio $(p<.05)$ y bajo $(p<.001)$; igualmente las diferencias entre los participantes de medio y bajo conocimiento son significativas $(p<.05)$.

En los conceptos concretos no se presentan diferencias significativas ( $p>$.05) en los contrastes realizados entre los tres grupos de participantes (figura 1 , sección b).

En los conceptos abstractos (figura 1, sección c) los participantes de conocimiento alto generan significativamente menos propiedades de entidad $(p<.001)$ que los de medio y bajo conocimiento. El contraste entre los porcentajes de los participantes de conocimiento medio y bajo son marginalmente significativos $(p=.057)$.

Diferencias por conocimiento. Todos los contrastes entre los porcentajes de propiedades de entidad de cada grupo de conocimiento para los tres conceptos arrojan diferencias significativas $(\mathrm{p} \leq .001)$. 


\section{Propiedades situacionales}

Las propiedades situacionales presentan efecto principal del factor concepto $(\mathrm{F}(2,135)=75.304, \mathrm{p}<$ $.001)$ e interacción concepto $\times$ conocimiento $(\mathrm{F}(4$, 272) $=9.944, \mathrm{p}<.001)$.

Diferencias entre conceptos. Los contrastes post hoc muestran que en los conceptos científicos los participantes de bajo conocimiento producen más propiedades situacionales que los de medio $(\mathrm{p}<.05)$ $y$ alto conocimiento $(\mathrm{p}<.001)$, respectivamente. Las diferencias entre los participantes de conocimiento medio y alto también son significativas $(\mathrm{p}<.05)$ (figura 1, sección a).

En los conceptos concretos no hay diferencias significativas entre los emparejamientos de los tres grupos $(p>.05)$ (figura 1 , sección $b$ ).

En los conceptos abstractos los participantes de conocimiento alto producen significativamente más propiedades situacionales que los de conocimiento medio $(p<.05)$ y bajo $(p<.001)$. Las diferencias entre los sujetos de conocimiento medio y bajo no son significativas $(p>0.5)$ (figura 1 , sección $c$ ).

Diferencias por conocimiento. Los participantes de conocimiento alto generan más propiedades situacionales en los conceptos abstractos que en los conceptos concretos $(p<.05)$ y en los científi$\cos (p<.001)$. A su vez producen más propiedades situacionales en los conceptos concretos que en los científicos $(p<.05)$.

Los participantes de conocimiento medio producen estadísticamente más propiedades situacionales en los conceptos abstractos y en los concretos que en los científicos $(p<.001)$. Sin embargo, las diferencias entre los pares de propiedades de los conceptos concretos y abstractos no son significativas ( $p>.05)$.

El porcentaje de propiedades de situación de los participantes de conocimiento bajo es mayor en los conceptos concretos que en los científicos $(p<.001)$. Los restantes emparejamientos no presentan diferencias significativas $(p>.05)$.

\section{Propiedades introspectivas}

El análisis de los datos de las propiedades introspectivas muestra el efecto principal del factor concepto $(\mathrm{F}(2,135)=247.632, \mathrm{p}<.001)$ e interacción concepto $\times$ conocimiento $(\mathrm{F}(4,272)=8.587, \mathrm{p}<.001)$.

Diferencias entre conceptos. En los conceptos científicos, las pruebas post hoc de los porcentajes de propiedades introspectivas evidencian que no hay diferencias significativas $(p=>.05)$ entre los tres grupos de conocimiento (figura 1 , sección $a$ ).

En los conceptos concretos los emparejamientos muestran diferencias significativas entre el porcentaje de propiedades introspectivas de los participantes de conocimiento alto y bajo $(p<.05)$. Los demás emparejamientos no presentan diferencias estadísticamente significativas $(p>.05)$ (figura 1 , sección $b$ ).

En los conceptos abstractos todos los emparejamientos intersujetos presentan diferencias significativas ( $p \leq .05)$ (figura 1 , sección $c$ ).

Diferencias por conocimiento. Los contrastes entre los porcentajes de propiedades introspectivas generadas por los participantes de conocimiento alto en los tres tipos de conceptos presentan diferencias significativas en todos los casos $(p \leq .05)$.

En los participantes de conocimiento medio, todos los emparejamientos entre los tres conceptos también presentan diferencias significativas $(p \leq .05)$.

En los participantes de conocimiento bajo, el porcentaje de propiedades introspectivas de los conceptos abstractos es significativamente mayor que en los concretos $(p<.001)$ y que en los científi$\cos (p<.001)$, respectivamente. La diferencia entre los conceptos científicos y concretos no es significativa $(\mathrm{p}>.05)$. 


\section{Discusión}

\section{Tipos de concepto}

El primer objetivo de este estudio era investigar el contenido conceptual de conceptos científicos con el de conceptos no científicos concretos y abstractos, para poder diferenciar su contenido.
Los resultados demuestran que el contenido conceptual de los tipos de conceptos estudiados es diferente. Aunque estadísticamente se presentan las diferencias en los conceptos en los niveles de conocimiento, es necesario analizar los resultados generales en cada concepto para lograr un panorama general en cada concepto. La figura 2 recoge los promedios porcentuales de cada propiedad en los tres conceptos.

Figura 2. Promedio de las propiedades en los tres conceptos (tomados de los promedios de la tabla 5)

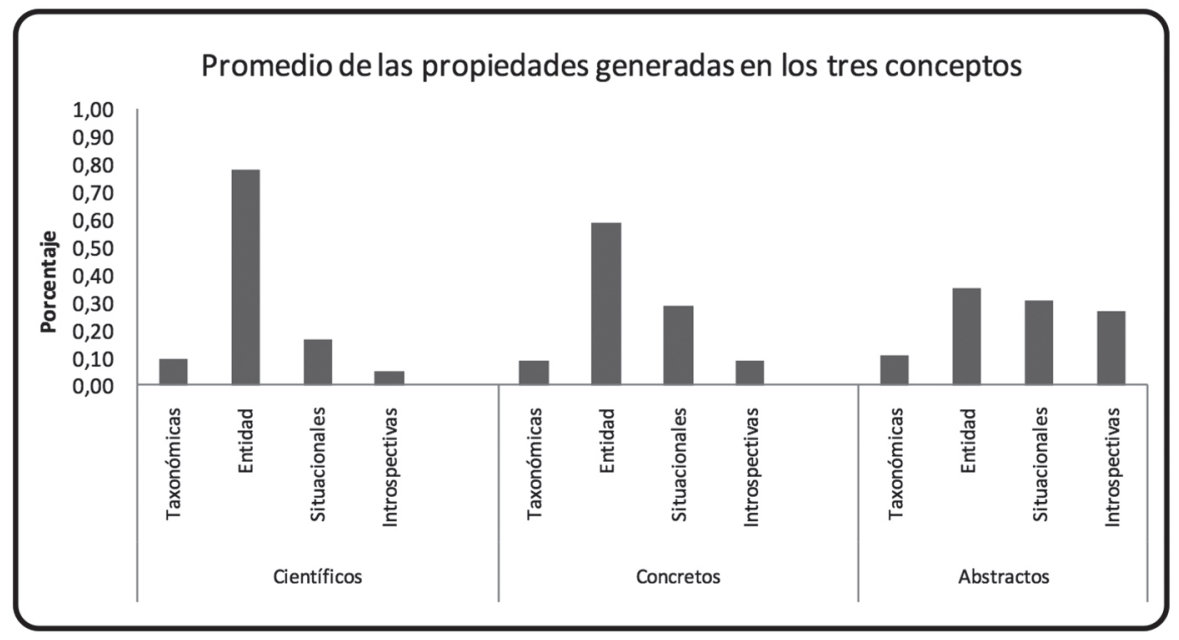

Los antecedentes sobre el contenido conceptual (Cree y McRae, 2003; McRae y Cree, 2002; McRae et al., 1997; Barsalou y Wiemer-Hastings, 2005; Wiemer-Hastings y Xu, 2005; Wu y Barsalou, 2009) sugerían que los conceptos concretos se centran más en la entidad que en las restantes, en tanto que los conceptos abstractos lo hacen más en los elementos situacionales e introspectivos. Los conceptos científicos, por su parte, muestran una alta concentración de su contenido en las propiedades de entidad.

Los resultados revelaron diferencias evidentes entre conceptos:

- En los conceptos científicos el foco está en la entidad (79 \%);

- En los conceptos concretos el foco también es en la entidad (59\%) pero se incrementan los elementos situacionales (28\%);
- En los conceptos abstractos el reparto es más equilibrado, no hay un foco definido (propiedades de entidad $35 \%$, situacionales $30 \%$, introspectivas $26 \%$ ).

Estos resultados son coherentes con la literatura que trata los conceptos concretos y abstractos. El contenido de los conceptos abstractos es más complejo que el de los concretos, puesto que no tiene un núcleo de contenido definido, como sí sucede en los conceptos concretos (Barsalou y WiemerHastings, 2005).

En lo que atañe al contenido de los conceptos científicos es de menor variedad que el de los concretos $y$, especialmente, que el de los abstractos. El conocimiento de los conceptos científicos almacenado en la memoria es fundamentalmente sobre la entidad. 
Las teorías corpóreas proponen que la conceptualización implica la intervención de conocimientos variados (p. ej. contexulaes) y no solamente los relativos a las entidades mismas. Sin embargo, los resultados indican que esta intervención de conocimientos que sitúan la simulación no es homogénea en todos los tipos de conceptos. En tanto los conceptos abstractos presentan un equilibrio entre los conocimientos sobre la entidad y los que sitúan dicha entidad, en los conceptos científicos los conocimientos situantes ocupan un lugar secundario. Los conceptos concretos se encuentran en un espacio intermedio de simulación. La suma de las propiedades situacionales e introspectivas en los conceptos abstractos alcanza el $56 \%$, en los conceptos concretos el $37 \%$, y en los conceptos científicos el $21 \%$. En contraste, como ya se apuntó, las propiedades de entidad en los conceptos científicos son el $79 \%$ del total, mientras que en los conceptos concretos son el $59 \%$ y en los abstractos el $35 \%$. La figura 3 muestra este contraste entre las propiedades de la entidad y las situantes.

Figura 3. Contraste entre las propiedades de entidad y las situantes. El porcentaje presentado como propiedades situadas es la suma de las situacionales e introspectivas en cada concepto.

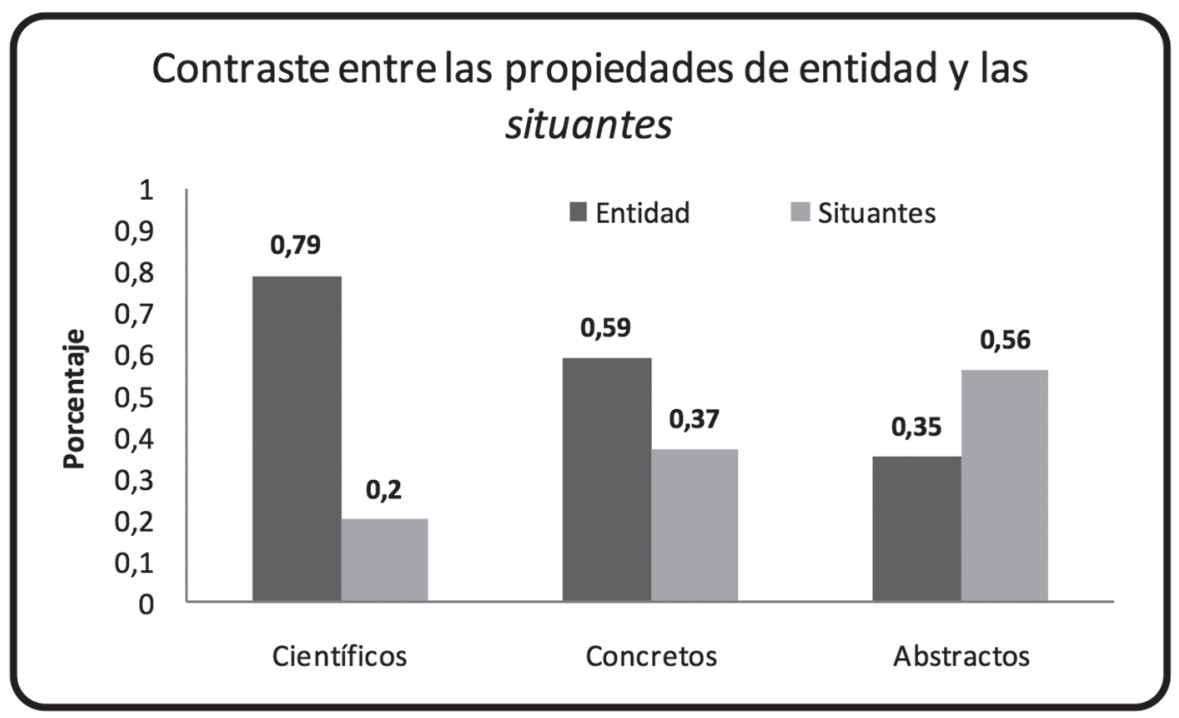

Ahora bien, también es preciso resaltar que de estos conocimientos situantes es en los conceptos abstractos donde más referencias introspectivas se generan. Mientras que las referencias situantes en los conceptos concretos y en los científicos son preferentemente situaciones y sus componentes, en los abstractos son experiencias y valoraciones subjetivas.

Por otro lado, y a pesar de estas diferencias en el contenido entre los conceptos, los resultados muestran que la ubicación taxonómica de los conceptos es semejante entre ellos. Al parecer no hay diferencias en la cantidad de información relativa a la pertenencia del concepto a una clase taxonómica. La proporción de este tipo de información oscila entre el 9 y el $11 \%$.
En consecuencia, mientras los conceptos presentan diferencias en cuanto a la cantidad de información de la entidad y la contextual, no las hay en cantidad de información taxonómica almacenada.

\section{El factor conocimiento}

El segundo propósito de este estudio consistía en investigar si la variable conocimiento influye en la simulación de los conceptos y, en consecuencia, en el contenido de los mismos. Específicamente, se esperaba que esta influencia se apreciara en los conceptos científicos puesto que el tipo de conocimiento que diferencia los tres conjuntos de participantes es el nivel de conocimiento en ciencias. 
Como ya se mencionó, en general en los conceptos científicos se presenta una mayor generación de propiedades de entidad. No obstante, se hallaron diferencias significativas entre los tres grupos de participantes (cfr. figura 1, sección a). Los participantes con mayor conocimiento en ciencias generan más propiedades de entidad que los otros dos grupos. Igualmente, los de conocimiento medio presentan más propiedades de entidad que los de bajo conocimiento. Estas diferencias permiten afirmar que se presenta una relación entre conocimiento y generación de propiedades de entidad en los conceptos científicos: a mayor conocimiento en ciencias mayor generación de propiedades de entidad.

De manera complementaria, también se evidencia que los grupos de participantes de bajo conocimiento generan más propiedades situacionales que los otros dos (medio y alto). Al parecer, en el desarrollo de la tarea de generación de propiedades, la "falta" de conocimiento no permite la concentración de la simulación en la entidad, y, en su lugar, se orienta hacia el contexto de la misma. Estos resultados sugieren, entonces, que al activar más conocimiento contextual y la experiencia subjetiva, las ideas intuitivas están más disponibles en la mente de los participantes de bajo conocimiento en ciencias. En este mismo sentido, parecería que la experticia, al permitir mayor concentración en la entidad, supone un énfasis en las relaciones de la entidad con sus propiedades y no con contextos corrientes.

Los resultados de los conceptos concretos, donde no se presentan diferencias significativas entre los tres grupos de participantes, permiten afirmar que la influencia del conocimiento específico se restringe a su dominio. El conocimiento en ciencias influye en la simulación de conceptos científicos, pero no el de conceptos de los cuales todos los participantes tendrían un conocimiento semejante (p. ej. árbol, sofá) (cfr. Figura 1, sección $b$ ).

No obstante, podría argüirse que los resultados de los conceptos abstractos (cfr. figura 1, sección c) no concuerdan con la influencia preferente del conocimiento sobre su área de experticia. Se hubiese esperado que los tres grupos de participantes tuvieran un comportamiento semejante, pues se suponía un grado de conocimiento semejante en los conceptos abstractos estudiados (p. ej. verdad, felicidad). Sin embargo, los participantes de conocimiento bajo en ciencias generan más propiedades de entidad que los otros dos grupos. La explicación más plausible radica en que estos participantes no son expertos en ciencias, pero sí poseen mayor acervo de conocimiento en humanidades, recuérdese que son estudiantes de humanidades (idiomas) y áreas a fines, lo cual implica mayor destreza lingüística en la descripción de este tipo de conceptos. En esta medida, los resultados de los conceptos abstractos antes que contradecir la influencia del conocimiento específico sobre su dominio, la confirma.

Los resultados de este estudio cobran valor no solo en el marco del conocimiento de los conceptos abordados, sino en el aprovechamiento educativo que se puede hacer de los mismos. Al parecer, el trabajo académico hace hincapié en las entidades mismas, es decir, en los objetos de estudio de cada disciplina. Sin embargo, hay otra porción de conocimiento almacenado sobre los conceptos que alude a su contexto. Reconocer el carácter situado de la cognición es una posibilidad educativa amplia pues la información que se activa en la mente es variada y no siempre centrada exclusivamente en los objetos de estudio, sino también en las situaciones físicas y mentales asociadas a los mismos.

\section{Referencias}

Achinstein, P. (1968). Concepts of science. A philosophical analysis. Baltimore: John Hopkins Press.

Barsalou, L. W. (1999). Perceptual symbol systems. Behavioral and Brain Sciences, 22(4), 577-660.

Barsalou, L. W., y Wiemer-Hastings, K. (2005). Situating abstract concepts. En D. Pecher y R. Zwaan (eds.), Grounding cognition: The role of perception and action in memory, language and thought (pp. 129-163). New York: Cambridge University Press.

Cree, G. S., y McRae, K. (2003). Analysing the factors underlying the structure and computation of the meaning of chipmunk, cherry, chisel, cheese, and 
cello (and many other such concrete nouns). Journal of Experimental Psychology: General, 132, 163-201.

Chi, M. (1992). Conceptual change within and across ontological categories: Examples from learning and discovery in science. En R. Giere (ed.), Cognitive models of science: Minnesota studies in philosophy of science. Minneapolis: University of Minnesota Press.

Chi, M. (2005). Common sense conceptions of emergent processes: Why some misconceptions are robust. The Journal of the Learning Sciences, 14(2), 161-199.

Chi, M. (2008). Three types of conceptual change: Belief revision, mental model transformation, and categorical shift. En S. Vosniadou (ed.), Handbook of research on conceptual change (pp. 61-82). Hillsdale, NJ: Erlbaum.

Chi, M., Slotta, J., y Leeuw, N. (1994). From things to processes: A theory of conceptual change for learning science concepts. Learning and Instruction, 4, 27-43.

Field, H. H. (2008). Saving truth from paradox. Oxford: Oxford University Press.

Giergyn, T. F. (1983). Boundary-Work and the Demarcation of Science from Non-Science: Strains and Interests in Professional Ideologies of Scientists. American Sociological Review, 48(6), 781-795.

Glenberg, A. M. (2007). Language and action: creating sensible combinations of ideas. En G. Gaskell (ed.), The Oxford handbook of psycholinguistics (pp.361370). Oxford, UK: Oxford University Press.

Glenberg, A., y Kaschak, M. (2002). Grounding language in action. Psychonomic Bulletin \& Review, 9(3), 558- 565

Glenberg, A. M., y Robertson, D. A. (2000). Symbol grounding and meaning: A comparison of highdimensional and embodied theories of meaning. Journal of Memory \& Language, 43, 379-401.

Hempel, C. G. (1952). Fundamentals of concepts formation in empirical science. Chicago: Chicago Press. [Versión en español: Fundamentos de la formación de conceptos en ciencia empírica. Madrid: Alianza Editorial, S.A. 1988].

Institut Universitari de Lingüística Aplicada, Universitat Pompeu Fabra (2005). Corpus 92. Recuperado de: http://www.iula.upf.edu/rec/corpus92/presenta.htm

Keil, F. C. (1989). Concepts, kinds, and cognitive development. Cambridge, MA: MIT Press.

Lawson, A. E., Alkhouty, S., Benford, R., Clark, B., y Falconer, K. A. (2000). What kinds of scientific con- cepts exist? Concept construction and intellectual development in college biology. Journal of research in science teaching, 37, 996-1018.

Mateus, G, y Otero, J. (2011). Memory content of scientific concepts in beginning university science students. Educational Psychology, 31(6), 675-690.

McRae, K., y Cree G. S. (2002). Factors underlying category-specific semantic deficits. En E. M. E. Forde y G. W. Humpreys (Eds.), Category-specifity in brain and mind (pp. 241-249). East Sussex, England: Psychology Press.

McRae, K., Cree, G., Seidenberg, M., y McNorgan, C. (2005). Semantic feature production norms for a large set of living and nonliving things. Behavior Research Methods, Instruments, \& Computers, 37(4), 547-559.

McRae, K., de Sa, V. R., y Seidenberg, M. S. (1997). On the nature and scope of featural representations of word meaning. Journal of Experimental Psychology: General, 126, 99-130.

Murphy, G. L., y Medin, D. L. (1985). The role of theories in conceptual coherence. Psychological Review, 92, 289-316.

Reif, F. (1987). Interpretation of scientific or mathematical concepts: Cognitive issues and instructional implications. Cognitive Science, 11, 395-416.

Reif, F., y Allen, S. (1992). Cognition for Interpreting Scientific Concepts: a Study of Acceleration. Cognition and Instruction, 9, 1-44.

Reiner, M., Slota, J. D., Chi, M., y Resnik, L. B. (2000). Naive Physics Reasoning: A commitment to substance-Based Conceptions. Cognition and Instruction, 18(1), 1-34.

Santos, A., Chaigneau, S., Simmons, W., y Barsalou, L. (2011). Property generation reflects word association and situated simulation. Language and Cognition, 3(1), 83-119.

Sebastián, G. N. (Coord.), Carreiras, M., Cuetos, F., y Martí, M. A. (2000). Lexesp: léxico informatizado del español. Barcelona: Universidad de Barcelona.

Suppe, F. (1973/1990). La estructura de las teorías científicas. Madrid: UNED.

Wartofsky, M. W. (1976). Introducción a la filosofía de la ciencia, I ( $2^{\text {a }}$ ed.). Madrid: Alianza Editorial, S. A.

Wiemer-Hastings, K., y Xu, X. (2005). Content differences for abstract and concrete concepts. Cognitive Science, 29, 719-736. 
Universidad Pedagógica Nacional

Facultad de Humanidades

Wu, L., y Barsalou, L. W. (2009). Perceptual simulation in conceptual combination: Evidence from property generation. Acta Psychologica, 132, 173-189.

Yeh, W., y Barsalou, L. W. (2006). The situated nature of concepts. American Journal of Psychology, 119, 349-384. 